\title{
Applicability of One-Diode Circuit Model to a PV Module in Real Environmental Conditions of Temperature, Air Mass, UV and Global Radiation
}

\author{
Santos, Hernani S. ${ }^{2}$, Santos, Lorena C. T.L. ${ }^{2}$, Silva, Pedro R. ${ }^{2}$, Batista, Ana P. ${ }^{1}$ and Jota, Patrícia R. S. ${ }^{1}$ \\ ${ }^{1}$ Department of Electrical Engineering \\ CEFET-MG \\ Campus II - Avenida Amazonas, 7675 -30510-000, Belo Horizonte (Brazil) \\ Phone/Fax number: +55 31 33196763, e-mail:prsjota@ cefetmg.br, anapaula@ des.cefetmg.br \\ ${ }^{2}$ Centro de Pesquisa em Energia Inteligente (CPEI) \\ Campus II - Avenida Amazonas, 7675 -30510-000, Belo Horizonte (Brazil) \\ Phone/ Fax number:+55 31 33196764, e-mail: hernanipace@ hotmail.com
}

\begin{abstract}
The prediction of energy converted into electricity by a solar panel is an important issue in the global context of expansion of the photovoltaic (PV) market. Although the general complexity of such predictions, PV systems boast in the simplicity of its modelling, made possible by means of the singlediode model. By this work a deeper comprehension of the relationship between the local solar ultraviolet (UV) radiation and the air mass (AM) with the current and power production of a PV panel, is achieved. The simple approach yielded by the model considers only datasheet parameters for forecasting the energy production of such a panel under the reference spectra, ASTM G173-03, which is not often found in the majority of the PV installations worldwide. Hence, the discussion provided by this paper aims to evaluate the influence of UV and AM on the energy production, and the possible usage of these variables on the referred single diode model.
\end{abstract}

\section{Keywords}

PV panel, one-diode model, spectral variations, air mass, UV radiation.

\section{Introduction}

The photovoltaic (PV) power generation is one of the main options on the market of renewable energy nowadays. During the years of 2000 to 2015 this technology has indeed exposed a prodigious growth of $42 \%$ in installations [1]. Moreover, this growth answers a global need, as the effects of human activity on earth is intensifying: the global warming, air pollution, damages to the ozone layer, and wild life mortality. Although the international awareness of the hazardous effects of the aforementioned issues [2], the global economy is still pushed by the possibilities of increasing profits and reduced payback time. It is actually triggering the development goals of PV technology to overcome the current low market participation and contribute to the optimistic scenario of $30 \%$ of the world power generation (until 2040) deriving from renewable sources [3].

Solar cells are responsible for the conversion of sunlight into electricity and Si-wafer based solar systems represented more than $75 \%$ of the total PV energy production in 2016, by two main technologies: monocrystalline and polycrystalline silicon, representing about $70 \%$ [1]. Both of them are designed considering STC (Standard Test Conditions), which is, reference conditions of: temperature, irradiation, AM and spectra.

Aiming a useful physic representation of solar cells, in the late decade of 1960 the single-diode equivalent circuit was developed for the modelling of PV devices, as an adaption of Shockley's considerations about carrier generation and recombination in P-N junctions [4]. Such considerations were well adapted to low complexity semiconductor devices, as single junction PV cells. Targeting a simplistic modelling, the referred equivalent circuit boasts the necessity of only the local irradiance and temperature as input variables for forecasting the production of a specific PV panel under STC. Thus, Figure 1 exposes the topology of the referred model [5]:

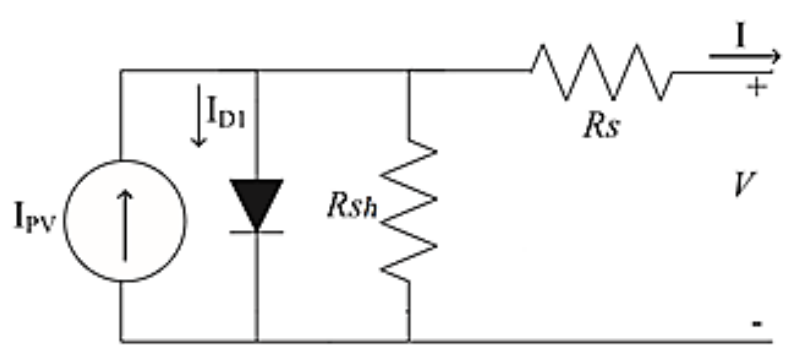

Fig. 1. Single-diode model of a solar cell. 
The current source represents the current produced depending on the incident light and local temperature. The diode in parallel with the current source addresses the recombination of carries in the quasi-neutral region. $\boldsymbol{R}_{\boldsymbol{S}}$ represents the mismatch characteristics of the semiconductor and contact resistances within the panel, while $\boldsymbol{R}_{\boldsymbol{s h}}$ quantifies a shunt resistance resultant of current deviations from the sides of the solar cell.

It has been reported that other external factors can change the current generation of the solar cell, for instance the AM level of a specific site during the measurements, which gives a good insight into spectral changes [6]. Hence, solar devices can be considered spectrally sensitive products, that require standard test conditions for allowing sharper comparisons between different technologies and devices. Thus, the standard spectrum ASTM G173-03 provides a reference spectrum that represent general clear sky atmospheric conditions, favourable for PV energy harvesting. The aforementioned standard considers a tilted surface with a $37^{\circ}$ slope of sun-facing and AM 1.5, a reasonable air mass value whereas the latitude characteristic of the majority of northern western countries.

Figure 2 presents main curves for the reference spectra.

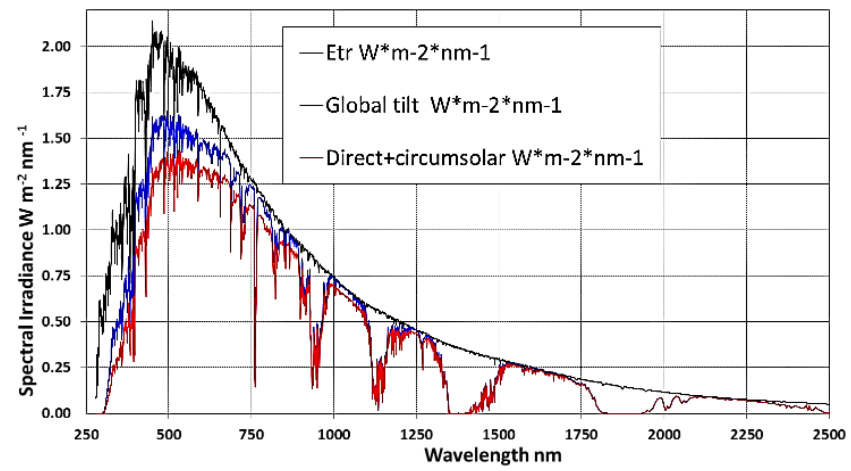

Fig. 2. ASTM G173-03 Reference Spectra

The spectral response of a silicon solar cell exposes its selective characteristic along the solar spectrum. In the UV interval (from 200 to $400 \mathrm{~nm}$ ), the Si-cell has a nonnegligible response, as shown in Figure 3:

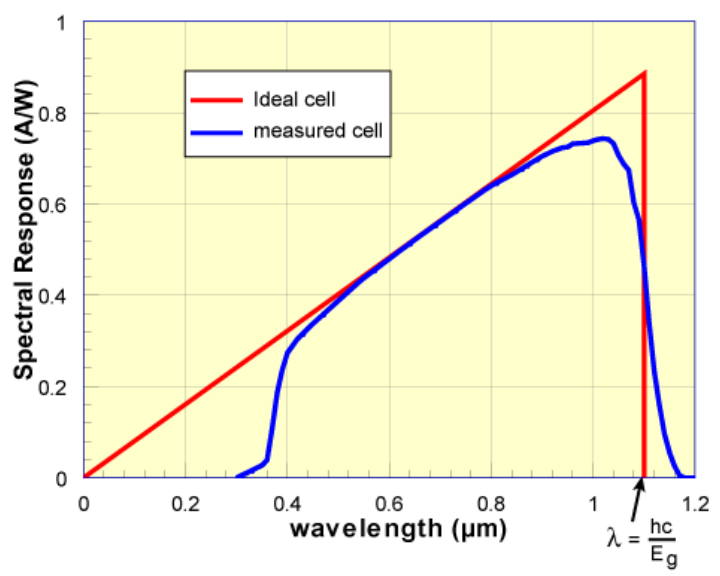

Fig. 3. Spectral Response Si-cell. Source: PvEducation (2017).
Previous works argued about this characteristic [7-9], discussing that the spectral variations bring changes on the produced short-circuit current, which reflects also on the overall efficiency of the device. Although these works have not clearly argued about introducing it on the PV cell circuit model, nor simultaneously relating specific variables such as AM and UV on the photocurrent generation.

In this paper, the modelling results of a PV panel, developed with a computational program based on the aforementioned 1-diode semi-empirical model, will be shown, considering different environmental conditions. Possessing the modelling and experimental data acquired during three autumn/winter months in Belo Horizonte, our analysis focuses on the influences of spectral variations on the production of a PV panel and its deviations from modelled data.

\section{Methods and Materials}

\section{A. Determination of the parameters of the equivalent circuit}

Considering the semi-empirical characteristic of the proposed model, an iterative procedure is necessary for the determination of its parameters. There are basically 5 parameters for the full applicability of the model, which are: $I_{P V}, I_{O}, R_{s h}, R_{S}$ and $a$, used in the equations (1)(3). Where, $I_{P V}$ is the photo-generated current, $I_{0}$ is the reverse saturation current, $R_{s h}$ is the shunt resistance, $R_{S}$ is the series resistance of the module and $a$ is the recombination coefficient of the diode, $\mathrm{D}$. The modelling process is shown by the flow chart found in Figure 4:

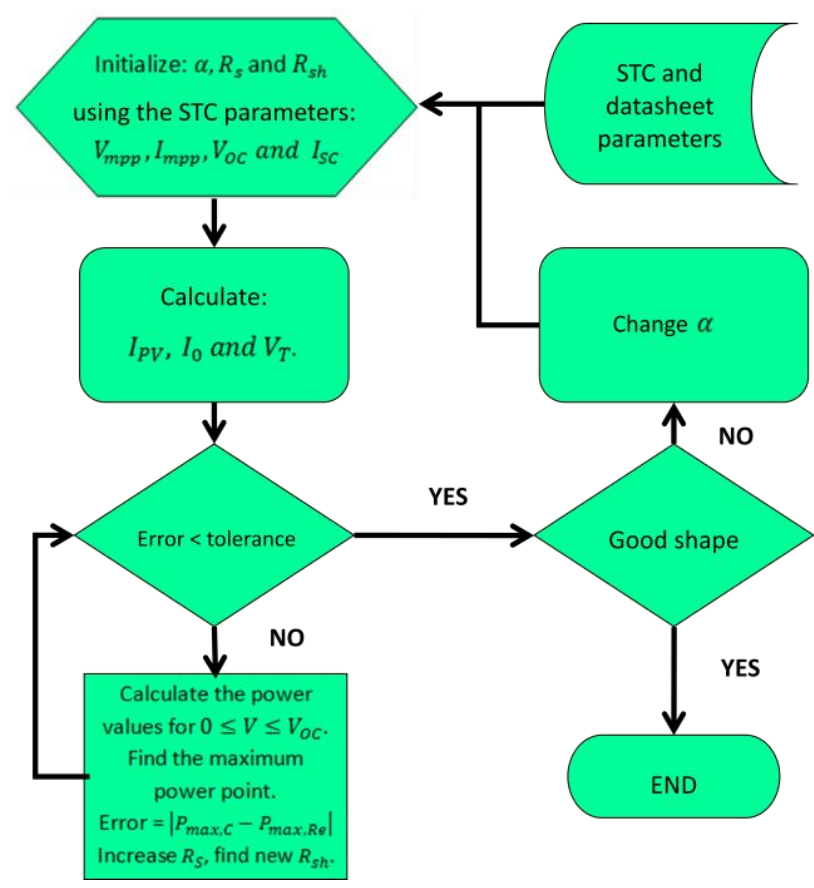

Fig. 4 - Flow chart of the modelling procedure. 
To initialize the iterative process, the initial values of the shunt, $R_{s h 0}$, and series, $R_{s 0}$, resistances are set as:

$R_{s h 0}=\frac{V_{m p p, S T C}}{I_{S C, S T C}-I_{m p p, S T C}}-\frac{V_{O C, S T C}-V_{m p p, S T C}}{I_{m p p, S T C}}, R_{s 0}=0 ;(1)$

Where, $V_{m p p, S T C}$ and $I_{m p p, S T C}$ are, respectively, the maximum power point voltage and current under STC. While, $V_{O C, S T C}$ and $I_{S C, S T C}$ are the open circuit voltage and the short circuit current under STC.

$$
I_{P V}=\frac{G}{G_{S T C}}\left(k_{I} \Delta T+I_{P V_{-} S T C}\right)
$$

As shown in equation (2), $I_{P V}$ is directly proportional to the irradiance, $\mathrm{G}$, incident on the cell, and to the local temperature. While the constant, $k_{I}$, which is the shortcircuit temperature constant, also influences the current production depending on the temperature. This current is, in other words, the photo-generated current, which is a function of the ratio of the irradiance, $G_{S T C}$, in the room and STC conditions, making it a fraction of the photocurrent under STC conditions, $I_{P V_{-} S T C}$.

Hence, the output current is expressed by:

$$
I=I_{P V}-I_{D}-\left(\frac{V+I R_{S}}{R_{s h}}\right)
$$

While $I_{D}$ is the recombination current expressed by:

$$
I_{D}=I_{O}\left[e^{\left(\frac{V+I R_{S}}{a V_{T}}\right)}-1\right]
$$

$I_{D}$, is almost insignificant in value in comparison to the photo-generated current. Many works have proposed the introduction of another diode in the model for representing the recombination in the depletion region also; however, the general results of this improvement generally are not justified by the increase in complexity that it requires. [10] - [12]

The voltage, $V_{T}$, is a temperature dependent parameter calculated by:

$$
V_{T}=\left(N_{s} k T \times a\right) / q
$$

Considering $T$ the local temperature, while $k$ is the Boltzmann constant $\left(1,3806485 \times 10^{-23} \mathrm{~m}^{2} \cdot \mathrm{kg} \cdot \mathrm{s}^{-2} \cdot \mathrm{K}^{-1}\right)$ and $q$ is the electron charge $\left(1,60217662 \times 10^{-19} C\right)$. Many authors have considered in their works $a$ between 1 and 2, depending on the fitting of the modelled curve in regard to the STC curves for the specific panel. [13] - [14]

After finding all these parameters, the expression for calculating $R_{s h}$ is found by simple circuit analysis:

$R_{S h}=\frac{V_{m p P, S T C}+I_{m p P, S T C} R_{S}}{\left\{I_{P V^{-}}-\frac{P_{m p p, E}}{V_{m p p, S T C}}-I_{O}\left[\exp \left(\frac{V_{m p p, S T C}+R_{S} I_{m p p, S T C}}{a V_{T}}\right)\right]\right\}}(5)$

\section{B. Experimental characterization system}

There are several topologies for obtaining the IxV and PxV curves of a photovoltaic module under test conditions, one of them is the simple and efficient capacitive load based circuit. [15]

This approach, shown in Figure 5, uses electronic switches, capacitors and resistors for representing the due load able to change from a short circuit unto an open circuit in a few seconds.

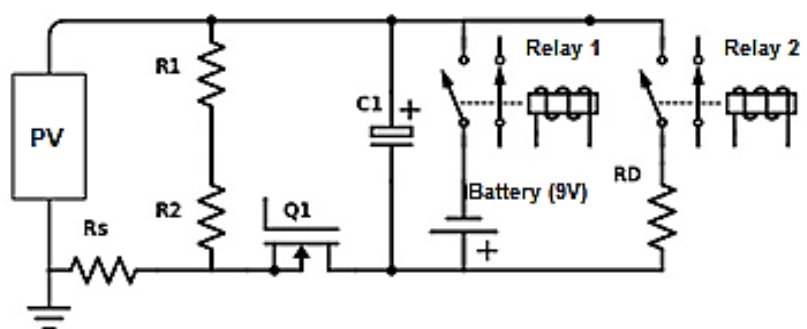

Fig. 5 - Capacitive load circuit for characterization of PV modules.

The PV device connected to the measurement circuit was the KC80, a 10-years old $80 \mathrm{~W}$ module left unexposed to sun light until our first measurements. The local radiation and other environmental conditions were acquired both by the weather station, Vantage Pro 2, and by photodiodes of UV and global radiation.

Measurements of AM were acquired by the positions $\log$ of a high accuracy bi-axial sun tracker system, built by EGIS. Figure 6 shows the installations and sensing devices in detail:

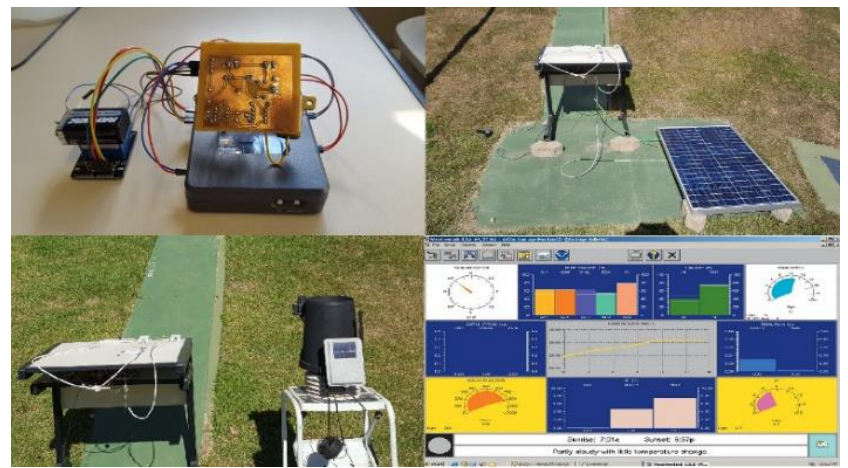

Fig. 6 - Installations of CPEI (Centro de Pesquisa em Energia Inteligente) to evaluate PV panels and weather conditions.

Finally, the statistical analysis was proceeded using StatGraphics, for the evaluation of the interaction among the main variables and their influence on the photogenerated current, as well. 


\section{Results and Discussion}

Below are the resulting parameters for the modelling of the referred panel:

TABLE I

STC SPECIFICATIONS FOR THE PANEL KC80 AND ITS PARAMETERS FOR THE PROPOSED 1-DIODE MODEL.

\begin{tabular}{cccc}
\hline Parameter & STC & Parameter & Model \\
\hline$V_{m p p}$ & $16,9 \mathrm{~V}$ & $V_{m p p}$ & $16,90 \mathrm{~V}$ \\
$I_{m p p}$ & $4.73 \mathrm{~A}$ & $I_{m p p}$ & $4.729 \mathrm{~A}$ \\
$V_{o c}$ & $21,5 \mathrm{~V}$ & $V_{o c}$ & $21,50 \mathrm{~V}$ \\
$I_{s c}$ & $4.97 \mathrm{~A}$ & $I_{s c}$ & $4.965 \mathrm{~A}$ \\
$N_{S}$ & 36 & $a$ & 1.1 \\
$K_{V}$ & $-8,24 \times 10^{-2} \mathrm{~V} /{ }^{\circ} \mathrm{C}$ & $R_{s h}$ & $236,98 \Omega$ \\
$K_{I}$ & $4,05 \times 10^{-3} \mathrm{~A} /{ }^{\circ} \mathrm{C}$ & $R_{S}$ & $0,24 \Omega$ \\
\hline
\end{tabular}

After adjusting the parameters, the model was validated in comparison to the datasheet curves under STC conditions.
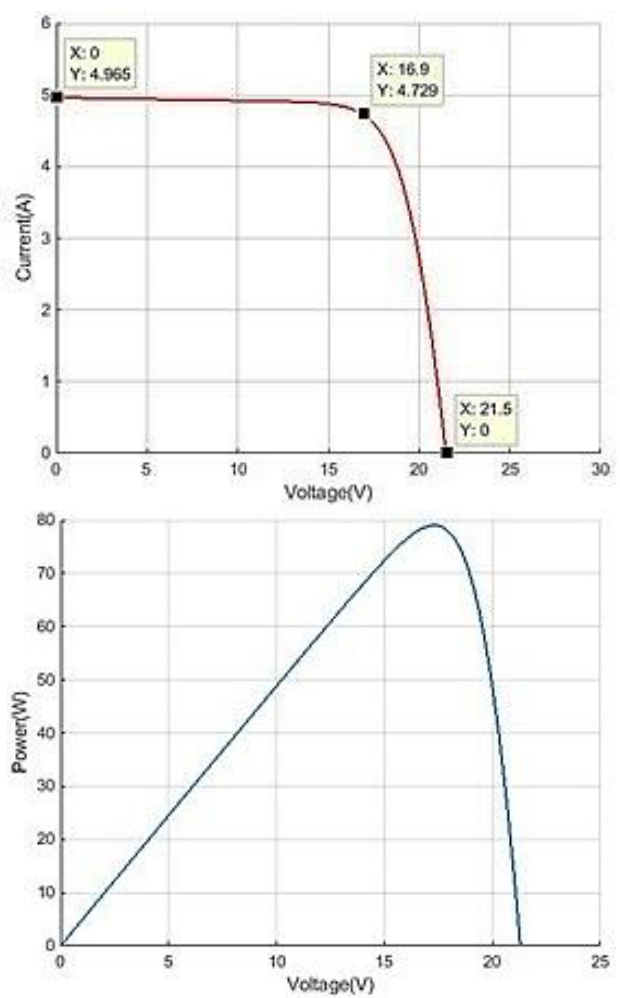

Fig. 7 - a) IxV and b) PxV modelled curves for STC conditions.

Comparing the data from Table 1 together with the curves in Figure7 it is possible to notice that the model was well adjusted for fitting IxV curves under fixed spectral conditions and variable irradiance and temperature levels.

After that, some tests were carried out for the comparison of the measured and modelled data for the panel during different days and hours for checking their compatibility. Figure 8 shows some of these curves:
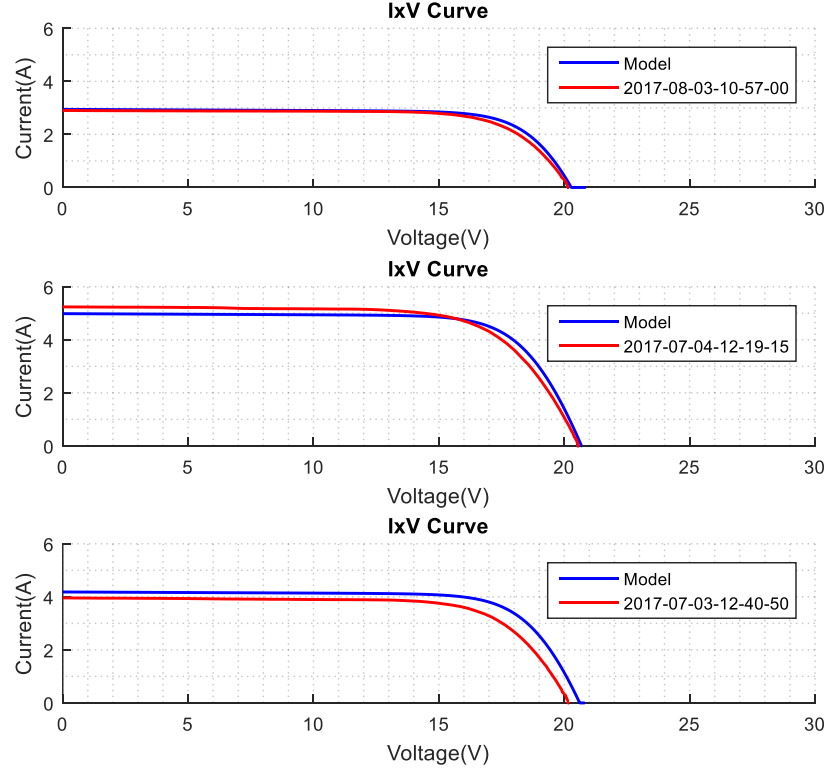

Fig. 8 - Comparisons of the modelled and experimental IxV curves for non-STC conditions.

By these tests, it was detected that in three different days there was a significant difference between the modelled and measured data, which was due to the spectral differences in comparison with the reference one. As mentioned before, the spectrum interferes in the efficiency of a PV device. Figure 9 presents a scatter chart relating the short circuit current with the UV level.

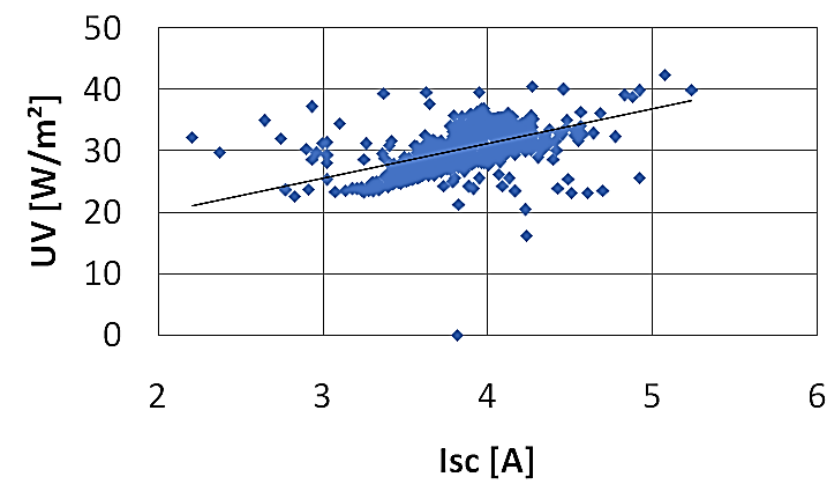

Fig. 9 - Scatter chart of UV radiation related to $I_{S C}$.

Although the correlation coefficient of 0,8959 and the visible tendency of proportionality between these variables, only this level of consideration is not enough for bringing assurance about it. Aiming to investigate the differences between the model and the real current production of the cell, it was made a set of analysis about the ratio between the measured short circuit current, $I_{s c \text {,meas }}$ and the modelled one, $I_{s c, \text { mod }}$. All of it under distinct AM, global radiation, and UV level. After that, all the data was filtered for a narrow interval of radiations, allowing a qualitative comprehension of the variability of the referred ratio in agreement with the AM and UV variations.

Applying the software StatGraphics for the factorial method, it was possible to evaluate the correlation between the input and output variables of interest in a quantitative manner focusing on the obtainance of deeper 
relations between the studied variables. Through this method it was obtained the ANOVA table:

TABELA II

THE ANOVA TABLE

\begin{tabular}{llll}
\hline Source & Sum of Squares & Mean Square & P-Value \\
\hline A:UV & 0,338526 & 0,338526 & $\mathbf{0 , 0 0 0 0}$ \\
B:Airmass & 0,0225996 & 0,0225996 & $\mathbf{0 , 0 0 0 0}$ \\
C:Radiation & 0,122851 & 0,122851 & $\mathbf{0 , 0 0 0 0}$ \\
AA & 0,534131 & 0,534131 & $\mathbf{0 , 0 0 0 0}$ \\
AB & 0,0116334 & 0,0116334 & $\mathbf{0 , 0 0 0 0}$ \\
AC & 0,121097 & 0,121097 & $\mathbf{0 , 0 0 0 0}$ \\
BB & 0,00190898 & 0,00190898 & 0,0533 \\
BC & 0,00145889 & 0,00145889 & 0,0911 \\
CC & 0,714757 & 0,714757 & $\mathbf{0 , 0 0 0 0}$ \\
Total error & 0,447165 & 0,000511046 & \\
Total (corr.) & 2,7578 & & \\
\hline
\end{tabular}

The ANOVA table, or analysis of variance, divides the output variability in separated parts for each one of the inputs, so this table exposes the statistical significance of the tested effects. The column P-Value shows that for the most influencing variable, the UV, the p-value was 0 , which confers warranty to the analysis using this variable.

Simultaneously to the ANOVA table it was obtained the Pareto's chart:

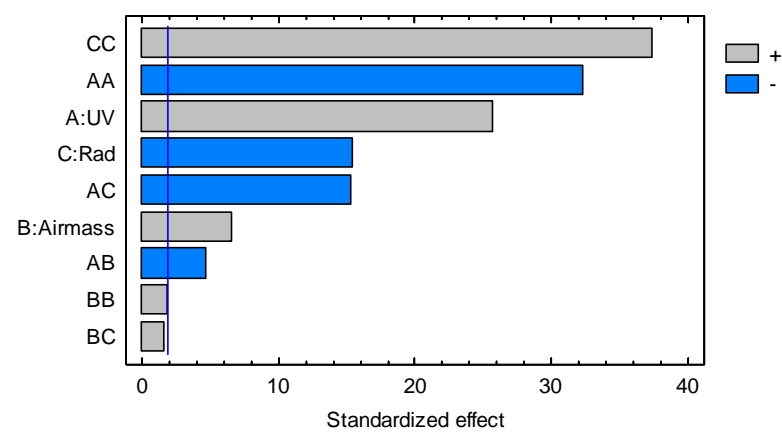

Fig. 10 - Standardized Pareto Chart for Isc ratio

The Pareto chart determines the most influencing factors on the output variable, that, in our case, is the ratio between the measured and modelled short circuit currents. In this chart, the positive and negative signals represent if the factor acts directly or indirectly on the output. And only the factors on the right of the threshold line, for instance the solar radiation and the UV level, are significant to change the output to generate influence on the photocurrent.

For a set of radiations close to $800 \mathrm{~W} / \mathrm{m}^{2}$, it was noticed a tendency of growth of the ratio following increases on the UV level, while when fixing also the UV and increasing the AM there was a tendency of reduction on the ratio. Cross variations of these two variables have revealed the preponderance of the UV on the changes of the ratio value in comparison with the AM.
Another useful chart for this analysis is the Main Effects plot, which follows bellow:

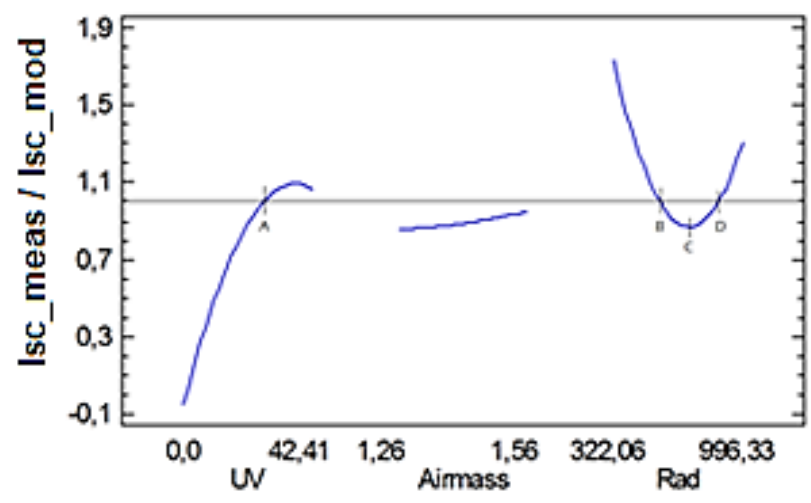

Fig. 11 - Main effects plot for the Isc ratio

By the chart of Figure11, it is possible to follow the growth of the main effect of UV in almost all the range analyzed until starting to present a decrease on the slope for values greater than $35 \mathrm{~W} / \mathrm{m}^{2}$. The AM effect is almost linear and has the lowest slope in comparison with the other two variables. The main effect of the solar radiation reveals a quadratic behaviour, with a decrease on the ratio for increasing radiations between the points $\mathrm{B}$ and $\mathrm{C}$ and a vertiginous growth for radiations increasing on the right side of $\mathrm{C}$. It reveals that the model calculates short circuit currents with values under the measured ones for the majority of the solar radiation range of the acquired data. After the analysis of the results from StatGraphics, some preliminary analysis were confirmed and other denied, showing that more trustful discussions about the performance of PV devices cannot be made considering only the effect of separated variables, such as AM, for instance.

\section{Conclusion}

In this paper, a deeper analysis on the applicability of the one-diode equivalent circuit for a PV panel under non STC conditions was proposed. Using the first calibrated model for the datasheet information on the forecast of production of a $80 \mathrm{~W}$ PV panel under Brazilian autumn/winter conditions, revealed mismatches between the modelled photocurrent and the measured one. After the first consideration of the preponderance of variables such as AM and UV as the individual cause of these mismatches, it was found by statistical analysis that, actually, the interaction of these two environmental variables with the radiation are crucial for a more accurate forecast of current production for PV panels at outdoor conditions.Since, although some differences between the modelled and the measured data seemed small for a $80 \mathrm{~W}$ solar panel, one must take into account the potentiality of this mismatch for a high power installation, such as a solar farm. Hence, future works could introduce this kind of relationships for tuning the referred model for more reliable modelling and energy production forecasts. 


\section{References}

[1] Fraunhofer, I. S. E. "Photovoltaics Report 12/07/2017." (2017).

[2] Clark, Peter U., et al. "Consequences of twenty-first-century policy for multi-millennial climate and sea-level change." Nature climate change 6.4 (2016): 360-369.

[3] Energy, I. E. A., and Air Pollution. "World Energy Outlook Special Report." Paris: International Energy Agency (2016).

[4] Sah, Chih-Tang, Robert N. Noyce, and William Shockley. "Carrier generation and recombination in pn junctions and pn junction characteristics." Proceedings of the IRE 45.9 (1957): 1228-1243.

[5] Duffie, John A., and William A. Beckman. "Solar engineering of thermal processes." (1980).

[6] Emery, Keith, Joseph DelCueto, and Willem Zaaiman. "Spectral corrections based on optical air mass." Photovoltaic Specialists Conference, 2002. Conference Record of the TwentyNinth IEEE. IEEE, 2002.

[7] Betts, Thomas R. Investigation of photovoltaic device operation under varying spectral conditions. Diss. (C) Thomas R. Betts, 2004

[8] De Soto, Widalys, S. A. Klein, and W. A. Beckman. "Improvement and validation of a model for photovoltaic array performance." Solar energy 80.1 (2006): 78-88.

[9] Hansen, Clifford. "Parameter estimation for single diode models of photovoltaic modules." Sandia National Laboratories, Albuquerque, NM, Forthcoming (2015).

[10] Charles, J-P., et al. "Consistency of the double exponential model with physical mechanisms of conduction for a solar cell under illumination." Journal of Physics D: Applied Physics18.11 (1985): 2261.

[11] Chin, Vun Jack, Zainal Salam, and Kashif Ishaque. "Cell modelling and model parameters estimation techniques for photovoltaic simulator application: A review." Applied Energy154 (2015): 500-519.

[12] Jordehi, A. Rezaee. "Parameter estimation of solar photovoltaic (PV) cells: a review." Renewable and Sustainable Energy Reviews 61 (2016): 354-371.

[13] Humada, Ali M., et al. "Solar cell parameters extraction based on single and double-diode models: A review." Renewable and Sustainable Energy Reviews 56 (2016): 494-509.

[14] Cárdenas, Alejandro Angulo, et al. "Experimental Parameter Extraction in the Single-Diode Photovoltaic Model via a Reduced-Space Search." IEEE Transactions on Industrial Electronics 64.2 (2017): 1468-1476.

[15] Muñoz, J., and E. Lorenzo. "Capacitive load based on IGBTs for on-site characterization of PV arrays." Solar Energy 80.11 (2006): 1489-1497. 